\title{
Best of ASCO 2021: new data from triple-negative breast cancer
}

\author{
Christian Singer
}

Received: 3 August 2021 / Accepted: 30 August 2021 / Published online: 4 October 2021

(C) The Author(s) 2021

\begin{abstract}
Summary This year's ASCO Annual Meeting has been a showcase for the overwhelming success of novel, targeted therapies, particularly in a tumor entity that has - until recently - been felt to be only treatable with chemotherapy. New data are extremely encouraging, but also highlight the need for target identification beyond the classical clinicopathological factors. Both, the Olympia and the Neotala study have been performed in BRCA-mutated tumors, and their results clearly point to the necessity to offer germline testing to HER2-negative high risk early breast cancer. In addition, GeparNuevo once more highlights the fact that immunotherapy is here to stay, not only in the advanced breast cancer setting, but also in early stage breast cancer. The side effect profile is acceptable, and long-term outcome a real improvement to conventional chemotherapy.
\end{abstract}

Keywords TNBC - BRCA · PARP inhibitor - Olaparib · Talazoparib

\section{OlympiA-olaparib in early high-risk gBRCA1/2- associated breast cancer}

The much-anticipated presentation of the results from the OlympiA trial certainly constituted the highlight of this year's ASCO conference. OlympiA is a phase III, multicenter, randomized, placebo-controlled trial of adjuvant olaparib after (neo)adjuvant chemotherapy in patients with germline BRCA1/2 mutations and high-risk HER2-negative early breast cancer [1]. The study investigates the efficacy and safety of oral PARP

\footnotetext{
Univ.-Prof. Dr. C. Singer, MPH ( $\bowtie)$

Klinisch-Translationelle Gynäkologische Onkologie, Brustgesundheitszentrum an der Medizinischen Universität Wien, Waehringer Guertel 18-20, 1090 Vienna, Austria christian.singer@meduniwien.ac.at
}

inhibitor (PARPi) olaparib in the early breast cancer setting. PARPi target cancers with homologous recombination repair defects by synthetic lethality. Olaparib is already licensed for platinum-sensitive ovarian cancer and for metastatic HER2-negative breast cancer with confirmed BRCA1/2 germline mutation. Women with a germline BRCAl/2 mutation who had developed HER2-negative (TNBC or hormone-receptor+ $[\mathrm{HR}+])$ high-risk early breast cancer were included after completion of their curative local treatment and (neo)adjuvant chemotherapy. Eligible patients with TNBC had $\geq \mathrm{pT} 2$ or $\geq \mathrm{pN} 1$ disease prior to adjuvant chemotherapy, or non-pCR (pathologic complete remission) after neoadjuvant chemotherapy; those with HR+ BC had $\geq 4$ positive nodes prior to adjuvant chemotherapy or had not achieved pCR. In addition, patients with non-PCR had to have a CPS\&EG (clinicopathological stage \& estrogen receptor expression and grading) score $\geq 3$. Patients were randomized 1:1 to receive either 1 year of continuous oral olaparib (300 mg BID) or placebo. Concomitant endocrine therapy and bisphosphonates were allowed. The primary endpoint of the OlympiA trial was invasive disease-free survival (IDFS) in the intention to treat (ITT) population. Secondary endpoints included distant DFS (DDFS), overall survival (OS) and safety. Safety analysis included adverse events of special interest (AESI; e.g., myelodysplastic syndrome/acute myeloid leukemia, new primary malignancy, pneumonitis). Overall, 1836 were enrolled between June 2014 and May 2019. Of these, $49.9 \%$ had received adjuvant chemotherapy, while $50.1 \%$ had undergone neoadjuvant chemotherapy. $26.5 \%$ of patients had received a platinum agent. Baseline demographics and tumor characteristics were balanced between the two study arms. After 165 IDFS events, a per-protocol IDMC interim analysis review was triggered which subsequently led to the IDMC recommendation to 
data unblinding. The subsequent analysis demonstrated a significant IDFS benefit of olaparib over placebo (hazard ratio [HR] 0.58; 99.5\% CI 0.41, 0.82; $P<0.0001)$ at 2.5 years median follow-up. IDFS events occurred in 106/921 and 178/915 patients assigned to olaparib and placebo, respectively. The 3-year IDFS was $85.9 \%$ vs. $77.1 \%$ (diff. $8.8 \%$; 95\% CI $4.5 \%$, $13.0 \%$ ). Similarly, DDFS was significantly improved with olaparib (HR 0.57; 99.5\% CI 0.39, 0.83; $P<0.0001$ ); 3-year DDFS was $87.5 \%$ vs $80.4 \%$ (diff. $7.1 \%$; $95 \%$ CI $3.0 \%$, $11.1 \%)$. OS was greater for olaparib, but was not statistically significant at the interim analysis (HR 0.68; $99.0 \%$ CI $0.44,1.05 ; P=0.024)$; 3-year OS\% $92.0 \%$ vs $88.3 \%$ (diff. $3.7 \%$; 95\% CI $0.3 \%, 7.1 \%$ ). Median intended olaparib exposure was $94.8 \%$, thus, suggesting good tolerability. Adverse events (AEs) were consistent with the label. G3+ AEs in $>1 \%$ of olaparib patients were anemia $(8.7 \%)$, neutropenia $(4.8 \%)$, leukopenia (3.0\%), fatigue $(1.8 \%)$, and lymphocytopenia (1.2\%). Serious adverse events (SAEs) were not increased by olaparib when compared to the placebo arm $(8.7 \%$ vs $8.4 \%$ ). The authors therefore concluded that adjuvant olaparib following (neo)adjuvant chemotherapy significantly improved IDFS and DDFS with acceptable toxicity in patients with gBRCAm and high-risk HER2negative EBC [1].

\section{NEOTALA - neoadjuvant talazoparib in gBRCA1/2-associated TNBC}

A second PARPi trial investigated the efficacy of neoadjuvant talazoparib monotherapy: the openlabel single-arm phase II NEOTALA trial study evaluated talazoparib at $1 \mathrm{mg} /$ day in 61 patients with stage I-III HER2-negative, ER/PR-negative breast cancer and germline BRCA1/2 mutations. Most women $(90 \%)$ received talazoparib for at least 20 weeks, and $74 \%$ received the drug for the full 24 -week study period. The primary endpoint was pathologic complete response rate by independent review in the evaluable population, which included patients who received at least $80 \%$ of the talazoparib dose and underwent surgery and assessment, plus patients who experienced disease progression before response could be assessed. Treatment with talazoparib resulted in pathologic complete responses in $45.8 \%$ of 48 evaluable patients and $49.2 \%$ of the intentionto-treat population of 61 patients. Event-free survival and overall survival had not been reached at the time of analysis. The investigators also looked at residual cancer burden (RCB). In the evaluable population, approximately $46 \%$ of patients had RCB0 and $31 \%$ had RCBII (moderate burden). No patients had RCBI (minimal burden) or RCBIII (extensive burden), and 23\% had RCB "other," which included patients who experienced disease progression on study and those who lacked surgery or assessment. Talazoparib was generally well tolerated, and treatment-emergent adverse events were consistent with the established safety profile, with mostly grade 1 or 2 fatigue, nausea, and alopecia. Grade 3 events were observed in $43 \%$; one grade 4 event occurred, which was decreased neutrophil count. Grade 3 anemia, a known toxicity associated with this drug, was observed in $39 \%$ of patients. The authors therefore concluded that talazoparib monotherapy was active in the neoadjuvant setting and showed pathologic complete response rates comparable to those historically observed with combination anthracycline- and taxane-based chemotherapy regimens [2].

\section{GeparNuevo-long-term outcome after durvalumab treatment in TNBC}

The German Breast Group presented the long-term outcome data of a neoadjuvant phase II study conducted in women with early breast cancer. In this trial, the addition of durvalumab, an anti-PD-L1 checkpoint inhibitor (CPI), was investigated when given to standard neoadjuvant chemotherapy in 174 patients with early TNBC, who were enrolled between June 2016 and September 2017. GeparNuevo randomized patients with cT1b-cT4a-d tumors and centrally confirmed TNBC to durvalumab $1.5 \mathrm{~g}$ i.v. or placebo every 4 weeks. Durvalumab/placebo monotherapy $(0.75 \mathrm{~g}$ i.v.) was given for the first 2 weeks (window phase), followed by durvalumab/placebo plus nab-paclitaxel $125 \mathrm{mg} / \mathrm{m}^{2}$ weekly for 12 weeks, followed by durvalumab/placebo plus epirubicin/cyclophosphamide (EC) q2 weeks for 4 cycles. Randomization was stratified by stromal tumor infiltrating lymphocytes (sTILs): low $(\leq 10 \%)$, intermediate $(11-59 \%)$, high $(\geq 60 \%)$. The primary objective was pCR (ypT0 ypN0). Secondary time-to-event endpoints included invasive diseasefree survival (iDFS), distant disease-free survival (DDFS) and overall survival (OS). The primary objective was reported previously and was less impressive: overall, durvalumab only offered a modest and nonsignificant improvement in pCR rates in response to durvalumab addition. There was, however, a small increase in pRR rates which appeared to be limited to patients treated with durvalumab alone before start of chemotherapy [3]. These results were, however, in striking contrast to the long-term outcome data, which were presented at this year's ASCO meeting: after a median follow-up of 42.2 months, 34 events occurred in 174 patients. The 3-year iDFS in pCR vs non-pCR was $92.0 \%$ vs $71.9 \%$ (log-rank $p=0.002$ ). The 3 -year iDFS was $84.9 \%$ with durvalumab vs $76.9 \%$ with placebo (HR 0.54, 95\%CI $0.27-1.09$, stratified log-rank $p=0.0559$ ); 3-year DDFS $91.4 \%$ vs $79.5 \%$ (HR $0.37,95 \%$ CI $0.15-0.87, p=0.0148$ ); 3 -year OS $95.1 \%$ vs $83.1 \%$ (HR 0.26 , 95\%CI $0.09-0.79, p=0.0076$ ). No difference was seen in iDFS, DDFS and OS between the window and no window cohort. The authors therefore conclude that durvalumab added to neoadjuvant chemotherapy in TNBC significantly improves long-term outcome despite a small pCR increase and 
no continuation after surgery. These phase II data need to be confirmed by a larger, phase III study which-among other research questions-needs to address the question whether adjuvant therapy with PD-L1 targeting drugs is needed at all [4].

\section{Take home message}

Data presented at ASCO 2021 now pave the way for the use of olaparib in early high-risk breast cancer in germline BRCA1/2 carriers. Encouraging results from the use of a PD-L1 antibody in the neoadjuvant treatment of TNBC allows improvement of long-term outcome and challenge our belief that improved long-term outcome is only limited to therapies that increase pCR rates.

Funding Open access funding provided by Medical University of Vienna.

Conflict of interest C. Singer reports financial and non-financial study support from Novartis, AstraZeneca, Sanofi, Daiichi-Sanyko, Amgen, Gilead, and travel grants and speaker's honorary from Roche, Novartis, AstraZeneca, Gilead Sciences, and Amgen.

Open Access This article is licensed under a Creative Commons Attribution 4.0 International License, which permits use, sharing, adaptation, distribution and reproduction in any medium or format, as long as you give appropriate credit to the original author(s) and the source, provide a link to the Creative Commons licence, and indicate if changes were made. The images or other third party material in this article are included in the article's Creative Commons licence, unless indicated otherwise in a credit line to the material. If material is not included in the article's Creative Commons licence and your intended use is not permitted by statutory regulation or exceeds the permitted use, you will need to obtain permission directly from the copyright holder. To view a copy of this licence, visit http://creativecommons.org/licenses/by/4.0/.

\section{References}

1. TuttANJ, Garber JE, Kaufman B, et al. Adjuvant Olaparib for Patients with BRCA1- or BRCA2-Mutated Breast Cancer. N EnglJ Med. 2021;384(25):2394-2405.

2. LittonJK, BeckJT,JonesJM, etal. Neoadjuvanttalazoparibin patients with germline BRCA1/2 mutation-positive HER2negative breast cancer: results of a phase 2 study. In: 2021 ASCO Annual Meeting. 2021. Abstract 505. Presented June 6.

3. Loibl S, Untch M, Burchardi N, Huober J, Sinn BV, Blohmer JU, etal. Arandomised phase II study investigating durvalumab in addition to an anthracycline taxane-based neoadjuvant therapy in early triple-negative breast cancer: clinical results and biomarker analysis of GeparNuevo study. Ann Oncol. 2019;30(8):1279-88. https://doi.org/10. 1093/annonc/mdz158.

4. Loibl S, Schneeweiss A, Huober JB, Braun M, Rey J, BlohmerJU,FurlanettoJ,ZahmDM,HanuschC, ThomallaJ, Jackisch C, Staib P, Link T, Rhiem K, Solbach C, Fasching PA, Burchardi N, Denkert C, Untch M. Durvalumab improves long-term outcome in TNBC: results from the phase II randomized GeparNUEVO study investigating neodjuvant durvalumab in addition to an anthracycline/taxane based neoadjuvant chemotherapy in early triple-negative breast cancer (TNBC). In: ASCO. 2021. Oral Presentation, Abstract 506.

Publisher's Note Springer Nature remains neutral with regard to jurisdictional claims in published maps and institutional affiliations.

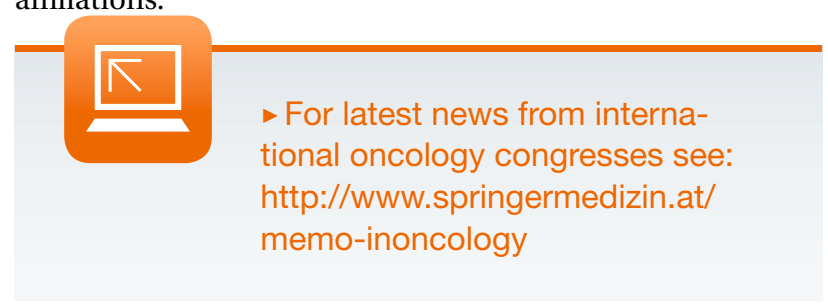

